\title{
The Temporal Automated System for Taste Experiments (TASTE)
}

\author{
AMIR ASHKENAZI \\ John B. Pierce Laboratory and Yale University, New Haven, Connecticut \\ MICHAEL FRITZ and JOHN BUCKLEY \\ John B. Pierce Laboratory, New Haven, Connecticut \\ and \\ LAWRENCE E. MARKS \\ John B. Pierce Laboratory and Yale University, New Haven, Connecticut
}

\begin{abstract}
We describe a new, open flow device for presenting taste stimuli to human subjects under controlled conditions of timing. The device delivers each stimulus as a mist to the participant's tongue through one of 16 nozzles attached to a linear slide. Software controls the position of the slide, the duration of the stimulus, and the duration of the pre- and poststimulus water rinses and records the responses of the participant. Temporal characteristics of this system make it especially applicable to studies on the role of attention in taste perception.
\end{abstract}

In this article, we describe a new device for presenting taste stimuli to human subjects under controlled conditions of timing, thereby making it possible to adapt a variety of popular experimental paradigms, such as cued attention, to the gustatory system. In many experiments on human taste perception, participants are required to sip a solution, hold it in their mouths for several seconds, spit out the solution, and then make a judgment. This judgment may involve detection (Marks \& Wheeler, 1998), discrimination (Laing, Link, Jinks, \& Hutchinson, 2002), intensity estimation (Rankin \& Marks, 2000), and the like. It is clear, however, that such a procedure is limited in several ways. For one, the method precludes precise standardization in stimulus presentation, in that the sip procedure makes it difficult to control the way the stimulus is taken into the mouth and, in particular, the location and duration of stimulation of the tongue and oral cavity. For another, the need to rinse away the taste solution and saliva before each trial strongly constrains not only the rate of stimulus trials, but also the capacity for imposing rapid changes in stimulation within a trial.

There are several ways in which the sip procedure precludes precise standardization of taste stimulation. First, because participants themselves control stimulus duration,

This work was supported by NIH Grant 5R01 DC00271-16 to L.E.M. We thank Tom D'Alessandro, Angelo DiRubba, and Ronald Goodman for assistance in constructing and setting up the system. We also thank Donald McBurney, an anonymous reviewer, and Jonathan Vaughan for their helpful comments on an earlier version of the article. Correspondence concerning this article should be addressed to A. Ashkenazi, John B. Pierce Laboratory, 290 Congress Ave., New Haven, CT 06519 (e-mail: aamir@jbpierce.org). duration may differ from participant to participant and thereby affect taste responsiveness (see, e.g., Bujas \& Ostojcic, 1939, cited by Marks, 1974). Second, even for a given individual, stimulus duration may vary from trial to trial, increasing the variability in stimulation and, hence, in response. Third, in the sip procedure participants also control the process of rinsing between stimuli, another possible source of both inter- and intraindividual variation.

The sip procedure constrains taste experimentation in other important ways as well. For instance, because participants control stimulus onset and offset, it is difficult to measure response times, a popular and important indicator of information processing. Temporal control is also important in studies of attention and constitutes a primary motivation for the present project. Marks and Wheeler (1998) did find, with a sip procedure, that attending to a particular taste stimulus, such as sucrose, increased its detectability relative to an unattended or unexpected stimulus, such as citric acid. However, the effects of attention were generally small, perhaps because the sip procedure gives subjects the opportunity to shift their attention to the unexpected stimulus within a trial. To control attention, stimulus duration should be fixed and relatively brief. This can be accomplished through the use of systems that flow taste solutions over the tongue for fixed periods of time.

The need for precise control over temporal properties of taste stimulation has, over the decades, led investigators to design various gustometers (Bujas, 1934, cited by Kelling \& Halpern, 1986; Pfaffmann, 1935), which Kelling and Halpern (1986) called closed and open flow delivery systems. In closed flow systems, the participant's tongue touches an opening in a tube (usually $<2 \mathrm{~cm}^{2}$ ) and the solution is delivered to a small portion of the tongue (Békésy, 
1963, 1964; Bujas, 1934, cited by Kelling \& Halpern, 1989; Bujas \& Ostojcic, 1939, cited by Marks, 1974; Hahn, 1949, cited in Bartoshuk, 1978; Hebhardt, Bagla, \& Doty, 1999; Kelling \& Halpern, 1983, 1987, 1988; McBurney, 1978). In open systems, the tongue is allowed to protrude from the mouth and the solution is flowed or sprayed on its anterior surface (Bonnet, Zamora, Buratti, \& Guirao, 1999; Guirao \& Zamora, 2000; Lester \& Halpern, 1979; McBurney, 1976; McBurney \& Pfaffmann, 1963). As has been noted, a critical property of a stimulus is its duration. Kelling and Halpern (1986) compared the actual stimulus duration (the time the stimulus flowed over the tongue) to its nominal duration in a closed flow system (Kelling \& Halpern, 1983) and an open flow system (Lester \& Halpern, 1979). Kelling and Halpern (1986) found actual stimulus durations to be significantly greater than nominal durations in the open system but not in the closed system.

Although this finding suggests that closed systems might be preferable to open systems, it is possible to calibrate the actual duration of stimulation in an open system. In this regard, McBurney (1976) produced a gustatory stimulus whose concentration could vary over time by a square wave function controlled in amplitude and temporal frequency. Furthermore, open systems have properties that can make them superior to closed systems. In particular, open systems can readily permit a relatively large number of different stimuli within an experiment, whereas closed systems are generally much more constrained. This is due to the fact that stimuli in a closed system are delivered through a single tube. Presenting multiple tastants requires thorough rinsing of the tube prior to every trial in order to prevent contamination from previous trials, greatly increasing the smallest possible interstimulus interval (ISI). This property of closed systems makes them far less useful than open systems in studies of attention, which commonly require sequences of brief cue and test stimuli with ISIs in the hundreds of milliseconds (see, e.g., Posner, 1978). Consequently, we designed and constructed an open flow system that could be particularly useful in studies of attention in taste.

\section{Description of the Taste Stimulator}

The Temporal Automated System for Taste Experiments (TASTE) aims to provide control over the timing of taste stimulation. ${ }^{1}$ The system has three main components: a set of pressure tanks, a bank of valves for switching solutions, and a user interface.

Pressure tanks (see Figure 1). Six 2.5-gallon paint tanks (Speedaire model PT281400AJ) were modified to store the stimuli during the course of the experiments. Each of five reservoirs holds three 1,000-ml glass bottles, which contain the taste solutions. The sixth reservoir holds a 3,000-ml glass bottle, which in our experiments generally contains deionized water for rinsing the tongue between stimuli (however, the contents of the reservoirs

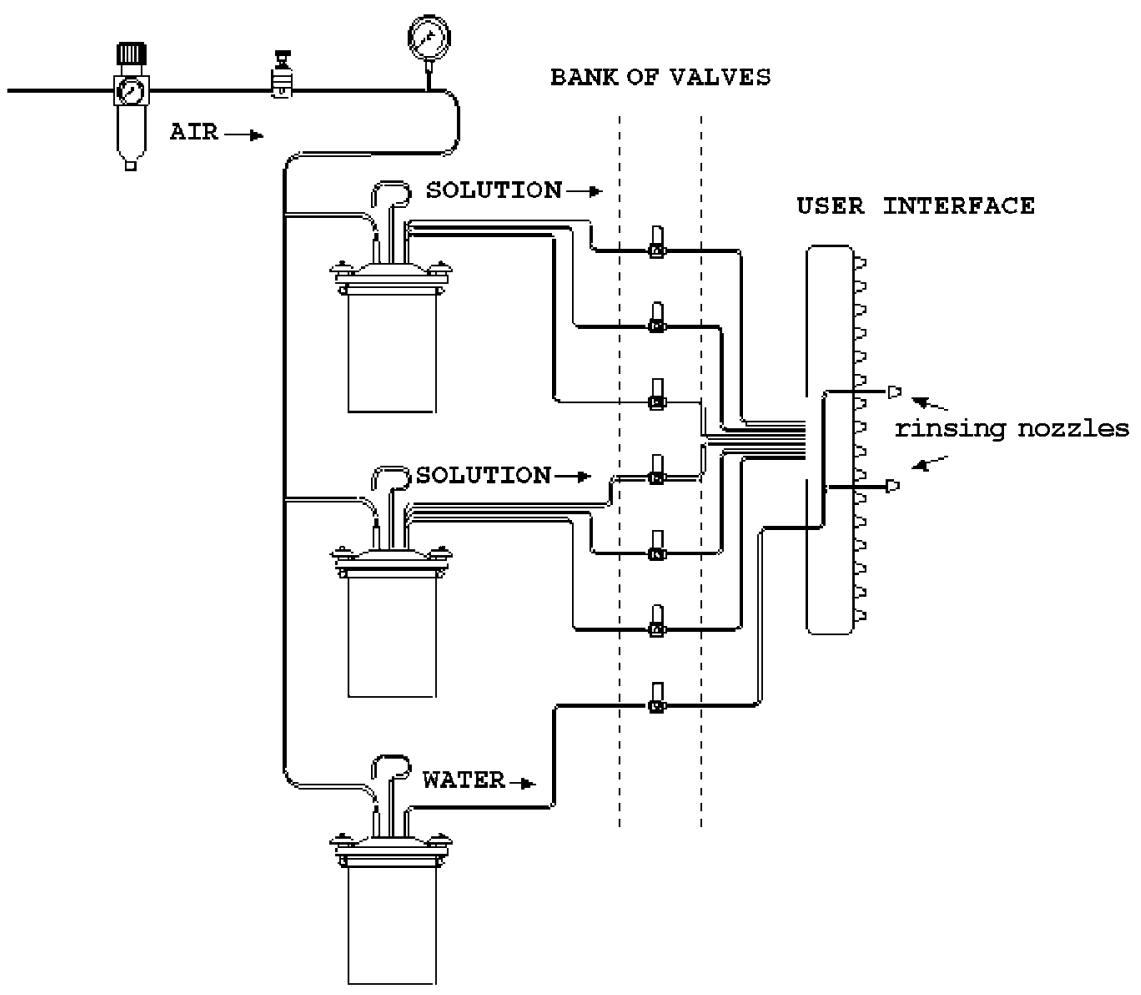

Figure 1. Diagram of the flow path of a single solution. 
can easily be modified to particular needs). To prevent corrosion, the inner side of each tank is covered with epoxy. A filter regulator assembly pressurizes the reservoirs up to $40 \mathrm{psi}$, and, to ensure safety, the reservoirs are placed within a closed metal box constructed of heavy-duty steel (1/16 in. thick). From each glass bottle within a reservoir, a Teflon PTFE tube (Parker/Atlantic; OD, 1/8 in.; wall, $1 / 32$ in.) delivers a solution to the bank of valves.

Bank of valves (see Figure 1). Each Teflon tube connects to a three-way isolation valve (NResearch Inc. model HP225T032). Each valve connects to a G4 digital output module (Opto22, Model G4ODC5), which, in turn, attaches to a G4 16-channel I/O module rack-isolated control (Opto22 Model G4PB16I). The I/O module serves to amplify the output current of a 48-bit high-drive digital I/O board for a PCI bus (Measurement Computing Corp., Middleboro, MA, Model PCI-DIO48H), whose operation is controlled through software written in Matlab (MathWorks, Inc., Natick, MA). On command, the valves open, enabling a solution to flow to the user interface. When closed, the pressurized solution in the tube releases to a sink, preventing dripping from the nozzles at the interface.

User interface (see Figure 2). Sixteen 1/8-in. NPT $80^{\circ}$ polyacetal misting nozzles (Tefen Mfg \& Plastics of Israel) are attached to a linear slide (Intelligent Actuator model DS-SA6-H-300). We selected misting nozzles in order to minimize tactile stimulation of the tongue, which might interact with taste perception (Cardello, 1981).

Following a signal from the computer, the slide can move from side to side and the preselected nozzle is placed in front of the participant's tongue. In addition, two rinsing nozzles, which are not attached to the linear slide, are located to the sides of the participant's tongue. This arrangement permits rinsing of the tongue while the linear slide is relocating, thereby minimizing the time between stimulus presentations.

Delivery of the stimuli is controlled through Matlab, which operates the three-way isolation valves, positions the linear slide, determines the duration of the stimuli, and records the participant's responses. Responses are made by pressing one of two response buttons. Thus, the hardware and software of the TASTE stimulator make the system compatible with various choice tasks (such as twoalternative forced-choice detection or discrimination) as well as simple tasks that require the use of only one response button. Finally, in the course of a trial, participants place their tongues on a disposable tube, which marks the place where the solution will be delivered (see Figure 2). To prevent delivery of the solution before the tongue is in place, participants have control over the initiation of the trial by pressing a foot pedal.

\section{Calibration of the Taste Stimulator}

Nominal stimulus duration is defined by Matlab software as the duration between the commands to open and to close a given valve. As we have already mentioned, however, actualduration, during which the stimulus flows from the nozzle, does not generally equal the nominal du-

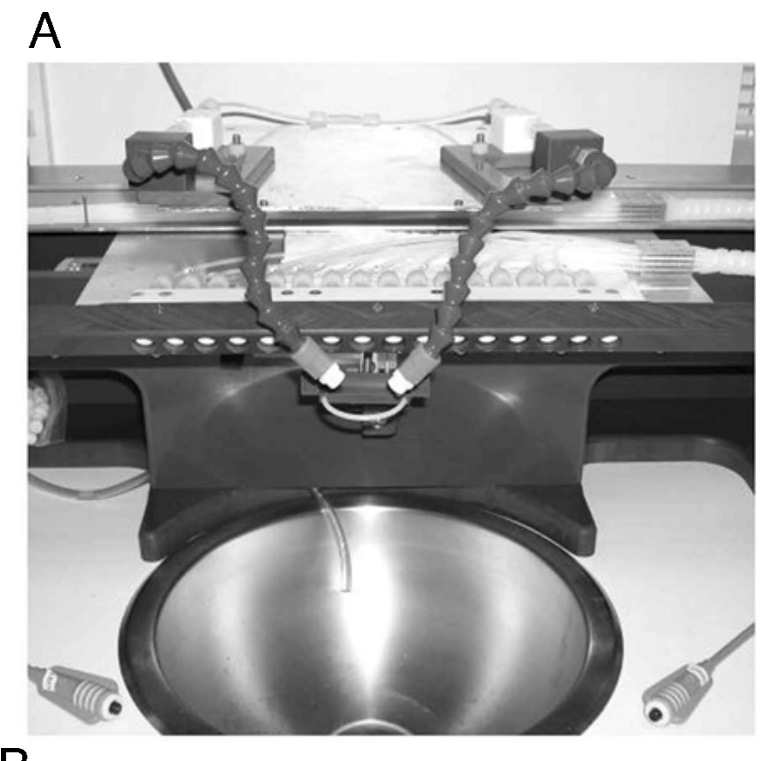

$\mathrm{B}$

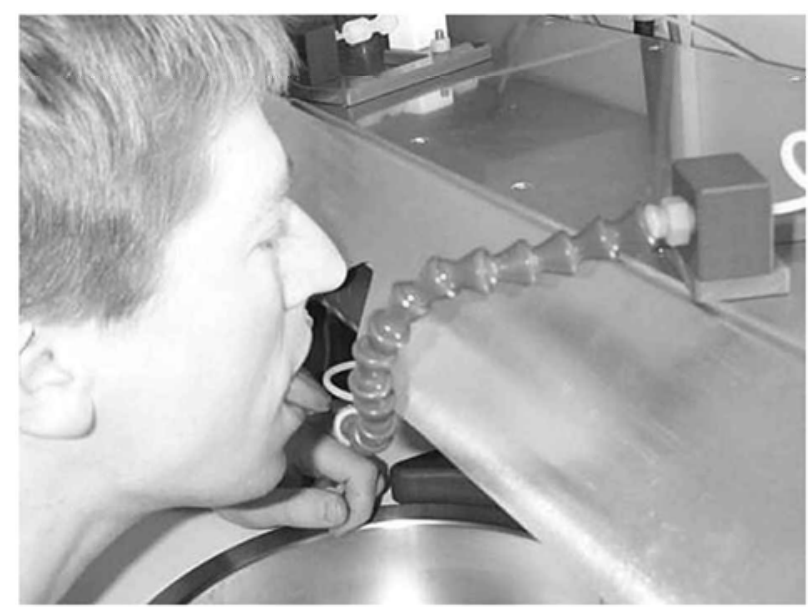

Figure 2. (A) The user interface section of the Temporal Automated System for Taste Experiments (TASTE). (B) The user interface of TASTE during an experiment. Note that the user interface is covered, preventing participants from seeing which nozzle is located in front of them.

ration. Actual duration exceeds nominal duration because of time lags resulting from passing commands from the computer to the valves, as well as from execution of the commands. Consequently, timing must be calibrated externally. To this end, we used a Thru-beam standard fiber set (SUNX Model FT-FM2) attached to a fiber-optic Thrubeam sensor (SUNX Model FX-301), a National Instruments A/D card, and a data acquisition program written in Matlab. The Thru-beam standard fiber set was placed as close as possible to the nozzles during the calibration phase to measure the actual stimulus duration.

We conducted calibrations on three measures: a 500msec stimulus duration, which represents a typical stimulus duration in future experiments; a shorter duration of $200 \mathrm{msec}$, which could be used in studies of attention; and 
the smallest ISI, which, in an attention task, may represent the interval between cue stimulus and test stimulus. Note that a 200-msec duration is fairly short relative to the stimulus duration typical in taste experiments, which is around 5 sec. This short duration, however, is useful in experiments that deal with attention. First, it prevents participants from shifting their attention to the unexpected stimulus within a trial. Second, it may help us to avoid adaptation to a long exposure, given that adaptation might overshadow the benefits of attention. Finally, a short stimulus duration makes it possible to present relatively large numbers of trials in a given experiment.

For the first two measures-durations of 500 and $200 \mathrm{msec}$ - we sampled the output from each nozzle on 20 repeated trials, with each duration initially set to the nominal values. The reading rate of the $A / D$ card was set to $1 / 200 \mathrm{sec}$ - that is, one reading every $5 \mathrm{msec}$. Figure 3 shows a time-reading sequence for typical trials. The variability observed is largely the result of the misting nature of the stimulus, which does not block the Thru-beam perfectly. For each nozzle, the average mist duration (Dm) was calculated, and a new nominal duration ( $\left.\mathrm{D}^{*}\right)$ was calculated by dividing the original nominal duration (Dn) by $\mathrm{Dm}$. The value of $\mathrm{D}^{*}$ was then set for each nozzle; these settings should produce actual stimulus durations of 500 (or 200) msec. This procedure of revising D* was repeated for each nozzle until we reached an average actual duration, Dm, between 490 and 510 (or between 190 and 210) msec. For the 500-msec durations, the overall average duration, pooled across nozzles, was $501 \mathrm{msec}($ range $=491-509$ ). The average standard error of the means was $10 \mathrm{msec}$ (range $=6-16$ ). For the 200-msec durations, the corresponding overall average duration was $200 \mathrm{msec}$ (range = 190-209). The average standard error of these means was $11 \mathrm{msec}$ (range $=6-23$ ). That the variability in these two measures is similar despite the difference in the means suggests that the variability reflects the physical limits (e.g., in switching) of the TASTE system.

We also measured the minimal ISI-that is, the smallest duration between two stimuli when these stimuli were delivered by the two distant nozzles. Twenty measures were taken for the sequence in one direction (Nozzle 1Nozzle 16) and 20 for the reverse sequence (Nozzle 16Nozzle 1). No difference was found in the average minimal ISI in the two sequences $(673$ and $660 \mathrm{msec}$, respectively). The overall average ISI was $667 \mathrm{msec}$ (range = 595-780). The standard error of the mean was $6.5 \mathrm{msec}$. Although an ISI of $500 \mathrm{msec}$ is relatively long for studies of attention, it is unlikely that it would be feasible to use shorter ISIs in taste experiments, given the need to rinse the tongue after each stimulus.

\section{Testing the Taste Stimulator}

Whereas the sip procedure delivers stimuli over much of the tongue and mouth, the TASTE system delivers stimuli to only a portion of the tongue. The difference in area extent of stimulation might matter to measures such as stimulus threshold or perceived intensity. As a practical

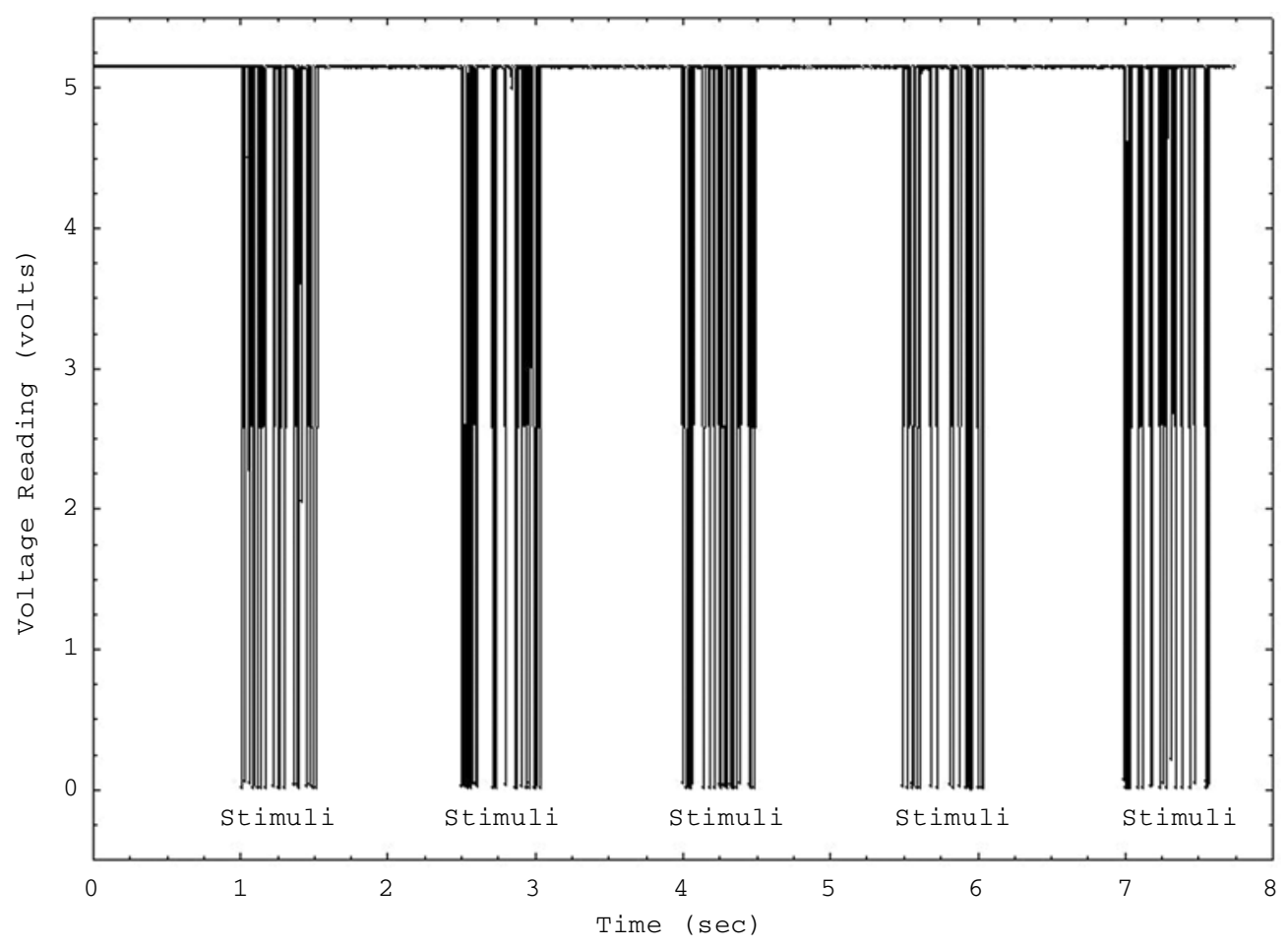

Figure 3. A time-reading sequence of five consecutive mists as measured by the Thru-beam calibration unit. 
matter, we need to ensure that participants can indeed detect solutions delivered over short durations through the TASTE system. Consequently, we made initial measurements of the detectability of weak sucrose solutions using an adaptive forced-choice method (the transformed updown procedure), with a three-down, one-up rule. This method was previously used by Marks and Wheeler (1998), with a sip-and-spit procedure under both baseline and selective-attention conditions. Thus, we are able to compare sucrose thresholds measured with the TASTE system with those measured by Marks and Wheeler using a comparable psychophysicalmethod in the baseline conditions of that study.

Two participants took part in the experiment. On each trial, the participants received two solutions in succession, one containing sucrose and the other, deionized water. The task was to indicate which solution contained the sucrose. Sucrose concentration increased on the trial following an incorrect response and decreased on the trial following three successive correct responses. This procedure enabled us to identify the concentration corresponding to a detection probability of .79. Sucrose concentrations ranged from -3.5 to $-1.0 \log$ molarity, in steps of $0.25 \mathrm{log}$ concentration units. The order of concentrations in the nozzle array was randomized for each participant. The initial concentration was the seventh level in the series, and the experiment continued until 13 transitions had been obtained, the last 6 of which were averaged to give the threshold. The duration of each stimulus was set to $500 \mathrm{msec}$.

The threshold for sucrose was $-1.67 \log$ molarity for 1 participant and $-1.91 \log$ molarity for the other. The present thresholds are about one order of magnitude greater than the corresponding average threshold of $-2.60 \mathrm{log}$ molarity reported by Marks and Wheeler (1998) using the sip-and-spit procedure. A higher threshold for the flow procedure in comparison with the sip-and-spit procedure is not surprising, given that the TASTE system stimulates a smaller portion of the tongue, over what is undoubtedly a shorter stimulus duration. There is considerable evidence of both spatial summation and temporal summation in taste perception (see, e.g., Linschoten \& Kroeze, 1991, 1992; Matsuda \& Doty, 1995). Given these considerations, the present data indicate that the TASTE system can be effective and useful in the study of gustatory perception.

It is important to note that although the TASTE stimulator can control stimulus duration to a considerable degree, like other open flow systems it provides little control over the region of the tongue stimulated. Furthermore, the use of a mist precludes accurate measurement of the spatial profile of stimulus concentration. Factors such as the angle and the distance between the nozzle and the tongue could contribute to variability between (and perhaps within) participants (see Figure 2b). This limitation, however, can be overcome by placing a sleeve on the tongue so that only the tip of the tongue, of a fixed area, would be stimulated. Such an adaptation could provide greater standardization of the region of the tongue stimulated-albeit at the cost, with a smaller area of stimulation, of lowering taste sensitivity even further.

\section{Summary}

In the present paper, we have described a system designed for delivering solutions containing taste stimuli for short, controlled durations and for switching relatively rapidly between tastants and water rinses. Although other devices exist for similar purposes, the open flow TASTE system allows presentation of stimulus sequences with relatively short ISIs. This is a crucial characteristic for experiments on such topics as the role of attention on taste perception.

\section{REFERENCES}

BARToshuk, L. M. (1978). Gustatory system. In R. B. Masterton (Ed.), Handbook of behavioral neurobiology: Vol 1. Sensory integration (pp. 503-567). New York: Plenum.

BÉKÉSY, G. VON (1963). Interaction of paired sensory stimuli and conduction in peripheral nerves. Journal of Applied Physiology, 18, 12761284.

BÉKÉSY, G. VON (1964). Rhythmical variations accompanying gustatory stimulation observed by means of localization phenomena. Journal of General Physiology, 47, 809-825.

Bonne t, C., Zamora, M. C., Buratti,F., \& Guirao, M. (1999). Group and individual gustatory reaction times and Piéron's law. Physiology \& Behavior, 66, 549-558.

BujAs, Z. (1934). Le temps d'action des stimuli de la sensibilité. Comptes Rendus des Séances de la Société de Biologie, 116, 1307 1309.

Bujas, Z., \& Ostojcic, A. (1939). L'évolution de la sensation gustative en fonction du temps d'excitation. Acta Instituti Psychologici, Universitatis Zagrebensis, 3, 3-24.

Cardello, A. V. (1981). Comparison of taste qualities elicited by tactile, electrical, and chemical stimulation of single human taste papillae. Perception \& Psychophysics, 29, 163-169.

GuiraO, M., \& ZAMORA, M. C. (2000). A computerized system for controlling and measuring gustatory reaction times. Journal of Sensory Studies, 15, 411-420.

HebHaRdT, P., BAgLA, R., \& Doty, R. L. (1999). An automated regional taste-testing system. Behavior Research Methods, Instruments, \& Computers, 31, 464-469.

Kelling, S. T., \& HAlPERN, B. P. (1983). Taste flashes: Reaction times, intensity, and quality. Science, 219, 412-414.

Kelling, S. T., \& Halpern, B. P. (1986). The physical characteristics of open flow and closed flow taste delivery apparatus. Chemical Senses, 11, 89-104.

Kelling, S. T., \& HAlPERn, B. P. (1987). Taste judgments and gustatory stimulus duration: Simple taste reaction times. Chemical Senses, 12, 543-562.

Kelling, S. T., \& Halpern, B. P. (1988). Taste judgments and gustatory stimulus duration: Taste quality, taste intensity, and reaction time. Chemical Senses, 13, 559-586.

LAing, D. G., Link, C., Jinks, A. L., \& Hutchinson, I. (2002). The limited capacity of humans to identify the components of taste mixtures and taste-odour mixtures. Perception, 31, 617-635.

Lester, B., \& HALPERN, B. P. (1979). Effect of stimulus presentation duration on gustatory reaction time. Physiology \& Behavior, 22, 319324.

Linschoten, M. R., \& Kroeze, J. H. (1991). Spatial summation in taste: $\mathrm{NaCl}$ thresholds and stimulated area on the anterior human tongue. Chemical Senses, 16, 219-224.

Linschoten, M. R., \& KROEZE, J. H. (1992). Bilateral taste stimulation: Spatial summation with weak $\mathrm{NaCl}$ stimuli. Chemical Senses, 17, 5359. 
MARKS, L. E. (1974). Sensory processes: The new psychophysics. New York: Academic Press.

Marks, L. E., \& WheEler, M. E. (1998). Attention and the detectability of weak taste stimuli. Chemical Senses, 23, 19-29.

MatsudA, T., \& Doty, R. L. (1995). Regional taste sensitivity to $\mathrm{NaCl}$ Relationship to subject age, tongue locus and area of stimulation. Chemical Senses, 20, 283-290.

MCBURNEY, D. H. (1976). Temporal properties of the human taste system. Sensory Processes, 1, 150-162.

MCBurney, D. H. (1978). Psychological dimensions and perceptual analyses of taste. In E. C. Carterette \& M. P. Friedman (Eds.), Handbook of perception VI(A): Tasting and smelling (pp. 125-155). New York: Academic Press.

McBurney, D. H., \& Pfaffmann, C. (1963). Gustatory adaptation to saliva and sodium chloride. Journal of Experimental Psychology, 65, 523-529.

PFAFFMANN, C. (1935). Apparatus and technique for gustatory experimentation. Journal of General Psychology, 12, 446-447.
Posner, M. I. (1978). Chronometric explorations of mind. Potomac, MD: Erlbaum.

RANKIN, K., \& MARKS, L. E. (2000). Chemosensory context effects: Role of perceived similarity and neural commonality. Chemical Senses, 25, 747-759.

\section{NOTE}

1. Owing to the complexity of the system, full structural and electronic schematics are not presented. Readers who would like to duplicate the TASTE stimulator are encouraged to contact Amir Ashkenazi (aamir@ jbpierce.org) or Lawrence E. Marks (marks@jbpierce.org) in order to obtain those schematics.

(Manuscript received January 16, 2003; revision accepted for publication October 5, 2003.) 